\title{
Les MOOC: du paradigme constructiviste à celui de la reliance
}

\section{MOOCs: From a constructivist approach to a reliant paradigm}

\author{
Florence G. Lojacono ${ }^{1}$ \\ Universidad de Las Palmas de Gran Canaria
}

\begin{abstract}
Much has been written about the MOOC phenomenon (Massive Online Open Courses), however, this is not an ICTE (Information and Communications Technology for Educational purposes) panegyric that we offer here. Instead, the objective is to make the most of these emergent educational modalities in order to start a reflection on the value of scientific criteria in educational research with a focus on French as Foreign Language (FFL). We will first show that many concepts seen as cutting edge educational innovations were actually imagined many years before. Therefore, we will see that it is only thanks to a favorable historical and technological context that the 2.0 education was born. This first point invites us to re-think constructivism, the dominant paradigm for research in education, and to investigate the pertinence of this central model within the knowledge society. In fact, characteristics of complex thought, as described by Edgar Morin, allow a more integrative, thus more realistic approach of the pedagogical equation and announce the possibility of a new paradigm: the "reliance" paradigm.
\end{abstract}

Keywords: constructivism, connectivism, complexity, paradigm, MOOC, FFE, Edgar Morin, reliance

1 Corresponding author - Universidad de Las Palmas de Gran Canaria, Departamento de Filología Moderna, Gran Canaria (Spain). Email: florence.lojacono@ulpgc.es 


\section{RÉSUMÉ}

Les MOOC (Massive Online Open Courses) ont déjà fait couler beaucoup d'encre et cet article ne vise pas à faire le panégyrique des TICE (Technologies de I'Information et de la Communication appliquées à l'Enseignement). II s'agit plutôt de prendre appui sur ces modalités éducatives émergentes afin de réfléchir sur le bien-fondé des critères scientifiques de la recherche en sciences de l'éducation, spécialement en FLE (Français Langue Étrangère). On montrera tout d'abord que bien des concepts actuellement présentés comme à la pointe de l'innovation pédagogique ont déjà été pensés il y a bien des années et que c'est uniquement grâce à un contexte social et technologique favorable que l'éducation $2.0 \mathrm{a} \mathrm{pu}$ voir le jour. Ce premier point invite à repenser le constructivisme, paradigme dominant en sciences de l'éducation, et à poser la question de sa pertinence dans le cadre actuel de la société de la connaissance. En effet, les caractéristiques de la pensée complexe telle que la décrit Edgar Morin permettent une prise en compte plus complète et donc plus réelle de l'équation pédagogique et annoncent la possibilité d'un nouveau paradigme, le paradigme de la reliance.

Mots-clés: constructivisme, connectivisme, complexité, paradigme, MOOC, FLE, Edgar Morin, reliance

\section{Introduction}

Cet article s'adresse à ceux et celles chargés de former les "Petites Poucette" ainsi que Michel Serres (2012) désigne ces jeunes qui commencent à arriver dans nos amphis, ces étudiants qui depuis leur tendre enfance ont usé de pratiques de lectures, d'écritures et de communications radicalement différentes de celles supposées par les manuels scolaires, de celles supposées par les grandes théories pédagogiques, qui balaient avec autant de régularité que d'inefficacité, le domaine de l'éducation. Les enfants de Petite Poucette bénéficieront sans doute de la plus grande transformation qui ait bouleversé le monde de la connaissance depuis l'invention de l'imprimerie. Au début de cette deuxième décennie du XXI ${ }^{\mathrm{e}}$ siècle, le déferlement ${ }^{2}$ des MOOC (Massive Online Open Courses) sur la place universitaire a provoqué une onde de choc dont on n'a pas fini de parler, et surtout, dont on n'a pas encore mesuré toutes les conséquences. Face au tout numérique on serait en droit de penser que le rôle du professeur, en tous cas tel qu'on l'envisage communément, est désormais superflu, ce qui dans certains milieux est d'ailleurs une crainte réelle. Loin de là. L'avènement de la connexion infinie, une des grandes mutations qui affecte l'éducation selon un rapport de l'OCDE de 2013, représente au contraire la chance d'un véritable retour aux sources de la relation pédagogique. Les yeux rivés sur nos écrans nous oublions

2 Dans son ouvrage au titre révélateur, Le tsunami numérique, Emmanuel Davidenkoff (2014) consacre d'ailleurs tout un chapitre au phénomène des MOOC. En 2012, le président de Stanford, John Hennessy avait déjà comparé l'arrivée des MOOC à un tsunami dans sa conférence The Coming Tsunami in Educational Technology. 
que, dans le domaine de l'enseignement, si la technologie a permis de revisiter et d'étendre l'audience des pratiques éducatives, les concepts eux-mêmes n'ont pas été dépassés par le tout numérique. Au XII ${ }^{\mathrm{e}}$ siècle par exemple, il y avait déjà des FAQ (Frequently Asked Questions en anglais, Foire Aux Questions en français) pour aider les étudiants nouveaux venus (Wieruszowski, 1966). Le numérique a certes facilité l'accès à ces FAQ mais n'en a pas inventé le concept. Le vocabulaire illustré imaginé par Comenius $^{3}$ (1592-1670) et les communautés d'apprenants conçues par Ivan Illich (1926-2002) sont encore au cœur de l'enseignement des langues. C'est de cela qu'il s'agit dans ces lignes: des possibilités d'une Renaissance de l'enseignement grâce au numérique et non d'un dithyrambe sur les prouesses des technologies de l'information et de la communication appliquée à l'enseignement (TICE). La réflexion proposée se compose de deux étapes qui se complètent. La première partie présente un état des lieux et prend en compte les principales peurs générées par l'universalité de l'accès au savoir, les $M O O C$ en étant le phénomène le plus représentatif. La seconde partie tentera de répondre à ces craintes, en insistant sur la non-nouveauté de concepts trop souvent présentés comme de purs produits des TICE et avec la nette volonté d'ouvrir le débat sur la pertinence d'un changement de paradigme dans les sciences de l'éducation.

\section{Ce numérique qui inquiète}

\subsection{Qui a peur du grand méchant MOOC?}

L'immense succès de la Khan Academy est un signe avant-coureur de ce point de nonretour qui fera basculer, dans un temps guère éloigné du nôtre, les enseignements traditionnels vers des formes à nous forcément encore inconnues mais que l'on peut déjà deviner. Fondée officiellement en 2008 par Salman Khan cette plateforme propose, gratuitement et à tous, des cours, des exercices et des parcours d'apprentissages avec un système de reconnaissance des savoirs acquis sous forme de badges. La Khan Academy ${ }^{4}$, spécialisée dans les disciplines scientifiques, enregistrait plus de 6 millions d'étudiants par mois en 2012 (Khan, 2013, p. 18). Nier l'importance du phénomène, de son impact sur les institutions éducatives et sur les pratiques cognitives serait adopter la politique de l'autruche. Offrir gratuitement des cours en ligne, sans distinction de publics, est aussi la caractéristique des MOOC, en

3 Consulter le livre de Comenius en ligne: <https://archive.org/details/johamoscommeniio00 come> [04/11/2015].

$4 \quad$ L'ONG Bibliothèques Sans Frontières traduit les vidéos ainsi que la plateforme Khan Academy en français, avec le soutien de la Fondation Orange et de nombreux bénévoles afin que les contenus soient accessibles dans le monde francophone. 
français CLOM (Cours en Ligne Ouvert et Massif) ou FLOT (Formation en Ligne Ouverte à Tous), dont Coursera, Udacity et Edx sont actuellement les fournisseurs les plus connus. À ces trois géants nord-américains se sont peu à peu ajoutés de nouveaux labels comme Canvas, puis l'Europe a suivi avec, par exemple, la création en 2013 de MiriadaX en Espagne et de FUN (France Université Numérique) en France. Si 2012 a été baptisée l'année des MOOC par le New York Times ${ }^{5}, 2013$ aura été celle des débats, parfois houleux, tant les MOOC soulèvent les passions. La polémique s'est installée en France à la suite de la parution de l'article de Libération du 26 décembre 2013 intitulé MOOC: une étape vers la privatisation des cours et de la réponse de Cyril Bedel publiée le 6 janvier 2014 dans le même quotidien. Le fait intéressant est que l'article du 26 décembre est l'œuvre d'un collectif anti-MOOC composé d'étudiants, de professeurs et de syndicats, ce qui laisse imaginer autant l'ampleur des enjeux que leurs ramifications politiques. De nombreux autres articles ont succédé, nous en citerons deux aux titres particulièrement évocateurs: Pièges à MOOC de Rachid Guerraoui (2014) et Moocs et vaches à lait d'Antoine Compagnon (2014). Ce dernier texte n'est disponible que moyennant paiement, ce qui est déjà, en soi, une réponse aux MOOC et à la libre circulation des connaissances. Si l'on en croit les titres quelque peu provocateurs des articles sur le sujet, il en ressort que l'usager des cours en ligne serait soit un être naïf soit un puissant imbécile. Ce verdict est-il compatible avec les milliers d'étudiants qui, tous les jours et depuis trois ans, s'initient ou se perfectionnent en algèbre grâce aux vidéos de la Khan Academy? Si certains avaient vaticiné pour 2014 la retombée de cet engouement (Rapp, 2014) les faits prouvent tout le contraire. En effet, FUN ${ }^{6}$ a annoncé en septembre 2015 avoir dépassé le million d'inscrits et la compagnie Orange s'est, elle aussi, lancée dans l'aventure en 2014 avec la plateforme Solemi spécialisée dans le B2B (business-to-business) et les COOP (Corporate Open Online Courses). Au même moment, à l'automne 2015, Telefónica Educación Digital, leader de la formation dans le monde hispanophone, lance de nombreuses solutions éducatives clé en mains dont deux portails destinés à la formation du XXI ${ }^{e}$ siècle. Le portail STEMbyme, similaire à la Khan Academy, propose des MOOC essentiellement scientifiques, avec un système de motivation sous forme de concours, de tournois et d'olympiades. Quant à ScolarTIC, ce portail rassemble une communauté d'enseignants et propose des ressources, des conseils et des formations reconnues grâce au système des badges. Mais même s'il ne s'agissait que d'une moocmania, n'en aurions-nous donc rien à apprendre?

5 Pappano, L. (2012). The Year of MOOC. The New York Times, 2 novembre 2012.

<http://www.nytimes.com/2012/11/04/education/edlife/massive-open-online-courses-aremultiplying-at-a-rapid-pace.html?_r=0> [04/11/2015].

$6 \quad$ <https://www.france-universite-numerique-mooc.fr/about> [20/01/2016]. 
L'ampleur de la controverse qui entoure les MOOC rend nécessaire une réflexion approfondie sur les bouleversements qui affectent l'enseignement en général, réflexion qui dépasse le cadre aride de toute polémique. Les craintes suscitées par les MOOC peuvent se lire comme la carte des faiblesses de l'enseignement traditionnel. Si les avis des uns et des autres sont si vigoureux c'est que les MOOC touchent le système éducatif là où cela fait mal: le changement radical et nécessaire du rôle de l'enseignant et la disparition possible des institutions éducatives traditionnelles. Que la figure de l'enseignant ait été constamment remodelée, soumise au bistouri des modes et au scalpel des politiques socio-éducatives, comme le suggère Philippe Mérieu dans son Frankenstein pédagogue (1996), et cela de manière de plus en plus précipitée au cours du xx ${ }^{\mathrm{e}}$, c'est un fait. On est en droit de se demander alors si l'ultime

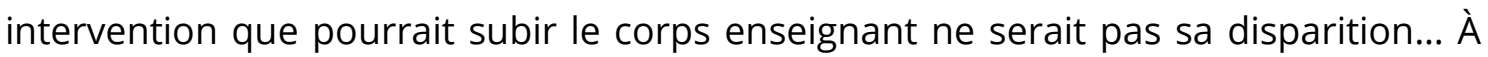
ceux qui craignent que le numérique ne fasse disparaitre les enseignants, on pourrait répondre que ceux-ci ont disparu depuis longtemps des précis de didactique des langues étrangères. Cela fait plus de trente ans déjà, que sous l'influence d'un courant venu d'outre-Atlantique, l'enseignant est devenu appreneur (Galisson, 1980, p. 51) personne-ressource, animateur, guide, conseiller, tuteur, médiateur ou facilitateur. Ce qui est repris et confirmé en 2002 par Cuq et Gruca (p. 139) qui assurent que l'enseignant qui se voit comme un transmetteur de savoir est démodé. Désormais adoubé organisateur-gestionnaire des enseignements il aura, bien sûr, des connaissances de la langue à enseigner mais il devra "avoir surtout un rôle de fournisseur de matériel" (Cuq et Gruca, 2002, p. 140). On comprend mieux alors la vigueur des débats autour des MOOC car si, en effet, telle est la fonction de l'enseignant-facilitateur, celui-ci ne tardera pas à être remplacé par une machine qui fournira plus de matériel, plus vite, sous des formes diverses et, de plus, $24 \mathrm{~h} / 24$, sept jours sur sept, sans rechigner et sans états d'âme.

\subsection{Magister vs Speaker?}

Serres écrivait que "jusqu'à ce matin compris, un enseignant, dans sa classe ou son amphi, délivrait un savoir qui en partie gisait déjà dans les livres" (2012, p. 35). Puisque le savoir est déjà écrit quelque part, et de plus facilement accessible, autant s'y approvisionner directement. Un enseignant "porte-voix" d'un savoir par d'autres conçus est, par conséquent, aussi obsolète qu'inutile. L'image du porte-voix reprend celle de l'enseignant speaker développée par Antoine Prost en 1985 dans Éloge des pédagogues. Trente ans plus tard, l'avis du CESE sur la pédagogie numérique (2015, p. 34) reprend, au sein d'une pédagogie constructiviste, l'opposition développée par Prost entre speaker et magister. Si le speaker ne fait que répéter ce que d'autres ont déjà dit/écrit/pensé sans se soucier de la réception de cette transmission d'informations, c'est au magister qu'échoie la noble mission de transmettre ce que lui 
seul peut transmettre efficacement: le traitement de l'information, l'organisation des connaissances et la méthodologie de travail. Dans cette métaphore du CESE c'est Internet, le speaker, qui est au service du magister. Cependant, que la technologie doivent être au service de l'humanité en général et de l'éducation en particulier, n'est pas un principe éducatif mais une question de bon sens comme le soulignait Célestin Freinet en 1946: "'instruction, comme les voies de communication, comme le téléphone et la radio, comme les machines nouvelles qui animent nos usines, n'est qu'un moyen, un outil. Tout dépend de l'esprit qui préside à son usage, du but pour lequel il est employé" (1978, p. 76). De plus, speaker et magister ne doivent pas forcément être réunis dans la même salle de classe, et c'est justement dans cette dissociation que réside la nouveauté pédagogique. Jose Bowen (2012) illustre de manière détaillée les usages d'une technologie utilisée au profit de la classe mais laissée en dehors de la classe, méthodologie appelée "la classe inversée" (flipped classroom en anglais et clase invertida en espagnol). Les étudiants arrivent préparés au cours grâce aux instructions précises de l'enseignant données en amont. Ces instructions font une large place à la communication synchrone et asynchrone, aux environnements virtuels d'enseignement, aux cours en ligne et aux plateformes multimédia. Le temps de classe est réservé aux questions, aux approfondissements, à la mise en pratique des connaissances et à la résolution de problèmes. Le fonctionnement de la classe inversée semble proche des conclusions du CESE: les technologies assument la fonction du speaker en dehors de la classe et l'enseignant celle du magister pendant la classe. II y a pourtant une différence fondamentale: dans le rapport du CESE c'est une relation d'exclusion qui s'établit entre magister et speaker. Loin d'intégrer les capacités du numérique pour refondre l'enseignement et redéfinir l'autorité des enseignants, on scinde les rôles. Cette vision frileuse n'a pas compris que, si en effet les savoirs et les processus cognitifs ont changé, ils se sont surtout complexifiés comment l'avait déjà souligné Edgar Morin en 2000 dans Les sept savoirs nécessaires à l'école du futur. Face à un enseignant du type "porte-voix" s'élève audessus des amphis un brouhaha permanent remarque Serres (2012), mais il en arrive de même face aux facilitateurs fournisseurs de matériel car Petite Poucette sait bien comment se procurer ce matériel toute seule et, de plus, sa façon à elle d'interagir avec les différentes sources et les divers formats donnera naissance à un matériel unique, plus riche et $100 \%$ personnalisé. Faire taire ce bavardage incessant demande une autorité que n'a pas l'enseignant porte-voix de connaissances désormais disponibles sur n'importe quel terminal connecté car, nous le disait déjà il y a vingt ans Francois de Closets, "à l'heure d'Internet, l'image du professeur omniscient est dépassée" (1996, p. 184). Nous n'assistons donc pas à la fin de l'ère du savoir mais à la fin d'une certaine façon d'envisager l'accès à ce savoir. C'est dans ces circonstances que la complexité doit être non seulement reconnue mais se situer au centre de la réflexion sur ce que signifie la transmission du savoir au Xxl ${ }^{\mathrm{e}}$ siècle. 


\section{Vers un changement de paradigme}

Les craintes dont nous venons de parler, et qui sont au cœur de la résistance antiMOOC, n'ont cependant de sens que si l'on considère avec Illich (1971, p. 88) que "le savoir est une marchandise qui, dans certaines conditions, doit être vendue de force au consommateur". Cette représentation de l'élève en "consommateur d'école" a d'ailleurs été reprise par le sociologue Robert Baillon en 1990. Une marchandise donc, dûment fabriquée, contrôlée, empaquetée et distribuée par des chaines de production certifiées ISO. Remarquons que les principales critiques faites aux MOOC, à savoir la privatisation des cours, la disparition des enseignants, voire celle des institutions, ainsi que l'uniformisation de l'offre académique à aucun moment n'envisagent de repenser les bases du système éducatif mais, bien au contraire, de les maintenir contre vents et marées. Dans les lignes qui suivent nous voudrions adopter, pour un court moment, un point de vue non marchand. II s'agit alors d'envisager le savoir libéré non seulement des chaînes de production qui le distribuent (i.e. les centres d'enseignement) mais libéré aussi de sa mise en scène appelée pédagogie. Nous nous apercevrons alors que la nouveauté radicale véhiculée par les MOOC, par l'idée d'une école grande comme le monde où la connaissance s'acquiert au long de parcours individuels aussi divers que variés, réside dans l'ouverture nécessaire des sciences de l'éducation à la pensée complexe.

\subsection{La pensée complexe}

Les TICE ne sont pas au cœur de la révolution éducative car les schèmes et les termes apparemment les plus novateurs de cette révolution ont déjà été pensés, et même expérimentés, il y a bien longtemps. Nous avons vu que les FAQ ne sont pas nées de cerveaux connectés à la fibre optique, et il en va de même du buzz, comme le démontre l'anecdote du pamphlet contre Louis XV en juillet 1749. Quand, après plusieurs semaines, on arrête l'auteur du coupable texte, celui-ci affirme, que s'il a bien eu le texte entre les mains il ne l'a pas écrit mais l'a reçu d'une autre personne. C'est le début d'une chaîne de coupables qui ne sont coupables que d'avoir passé le message, non de l'avoir écrit. En moins d'un mois il y a tant et tant de "messagers" que la piste de l'auteur est définitivement perdue: "la police du roi cherchait une personne, elle a trouvé un réseau social" (Fogel et Patino, 2014, p. 11). Mais l'analogie avec nos media hyper et inter connectés va encore plus loin. Le texte du pamphlet ne reste pas le même au cours de ces différents passages, il s'est modifié, il s'est pluralisé. Non seulement l'auteur n'a pas été trouvé mais la police n'est pas même certaine de détenir la version définitive du texte incriminé. Cette diffusion est appelée de nos jours un buzz. À l'ère d'Internet le pamphlet de 1749 aurait été qualifié de "viral" et ses différentes et insaisissables versions de "mèmes", un mème étant un "contenu cité, copié, détourné, diffusé de façon rapide" (Fogel et Patino, 2014, p. 135). C'est le 
contexte qui diffère, non la pensée. Les TICE ne sont le moteur d'aucune véritable révolution pédagogique mais en sont l'indispensable catalyseur dans un contexte social favorable.

Prenons l'exemple de l'enseignement du Français Langue Étrangère (FLE). Des années 1980 à nos jours, l'ère constructiviste inaugurée par Piaget règne sans partage. Ces conceptions, devenues socioculturelles, "puisent dans l'idée vygotskienne originelle selon laquelle l'activité humaine ne peut pas être considérée comme indépendante de son environnement social et culturel" (Zourou, 2007, p. 4) et sont clairement désignées comme formant un paradigme. Si le constructivisme est depuis les années 1930 un paradigme épistémologique à la suite des travaux de Bachelard et de Piaget (Le Moigne, 2012), il s'est par la suite fossilisé en paradigme idéologique de la recherche en FLE et c'est en tant quel tel que nous l'abordons ici. Cette fossilisation a empêché que de nouveaux paramètres soient pris en compte au sein d'une refonte générale de la recherche en sciences de l'éducation. En matière de pédagogie, on s'est longtemps contenté d'une pensée binaire: hier le maître sur son estrade face à des pupitres bien alignés, aujourd'hui la personne-ressource au milieu d'une classe en $U$ (Lojacono, 2009). Ce que les TICE pointent de leurs souris, c'est la fin de cette pensée binaire, c'est le fait qu'il faudra bien finir par s'atteler à une vraie réflexion et remettre en cause, non la pertinence passée, mais l'efficacité future du paradigme constructiviste. Ce n'est pas avec les tablettes et autres téléphones intelligents que la complexité est entrée dans nos salles de classe mais avec les étudiants issus de la génération connectée, de la génération du travail collaboratif, en somme de ce qu'on appelle la génération $C$. L'enseignant d'aujourd'hui doit faire face aux besoins d'un public dont les processus cognitifs lui sont le plus souvent complétement inconnus. Deux exemples qui en disent long: le tableau de feutre, innovation des années 70, est encore en 2002 rangé dans les supports technologiques du Cours de didactique du français langue étrangère et seconde de Cuq et Gruca (p. 421) et le minitel, que plus personne n'utilise en France depuis la fin des années 90 est pourtant présenté, en 2007, comme exemple "de canevas narratifs en temps réel et à distance" dans L'approche par compétences dans l'enseignement des langues de Jean-Claude Beacco (2007, p. 243). Les dates de publication recouvrent parfois d'un voile pudique l'obsolescence des contenus. Ce n'est pas seulement l'ingénierie éducative qui a évolué, c'est aussi et surtout la perspective choisie pour tenter de décrire, et donc de mieux comprendre, ce qui se passe entre un enseignant, un apprenant et un environnement multimédia. En effet, ces trente dernières années la pensée binaire a eu la main mise sur la pédagogie du FLE. C'est Robert Galisson en 1980 (p. 48) qui explique que le passage de "la prédominance de l'enseignant à la prééminence de l'apprenant" est une caractéristique de la didactique, c'est Beacco en 2007 (p. 37) qui fait la distinction entre une approche communicative "forte" ou "haute" et sa version désagrégée "faible" ou "basse". Nous avons vu plus haut de quelle façon l'opposition 
entre magister et speaker de 1980 a été reprise sans sourciller en 2015. Continuer sur ce chemin ne va pas aider la pédagogie à sortir de l'ornière où elle s'enlise depuis des lustres. Longtemps la pensée pédagogique ne s'est occupée que de renverser les rôles, rôles d'ailleurs très caricaturés afin d'en expurger tout ce qui n'entrait pas dans le moule. Et c'est justement à ce qui n'entre pas dans le moule, à ce qui a été volontairement omis par une pensée réductionniste, que va s'attaquer la pensée complexe. La pensée binaire exclut les paramètres qu'elle ne peut ou ne veut prendre en compte dans une équation incomplète qui par conséquent reflète un réel simplifié à outrance. Elle "ne voit que l'alternative ou/ou et se révèle incapable de combiner la conjonction et/et" (Morin, 2015, p. 37). Selon les auteurs de manuels la pensée binaire est supposée aider l'enseignant à distinguer le bien du mal et, pour cela, elle lui propose ses cadres bien clairs dont tous les termes s'opposent deux à deux.

Mais l'enseignant n'est pas seul face à ses élèves, entre eux il y a un médiateur obligé, imposé par le marché de l'édition autant que désiré par les acteurs des institutions éducatives: le manuel, support papier ou disponible en ligne. Voyons à présent quels sont les choix théoriques qui sous-tendent la conception pédagogique de ces manuels. Après l'approche communicative des années 80 , la dernière mode pédagogique est l'approche actionnelle, décrite dans un texte officiel à retombée internationale, le Cadre Européen Commun de Référence pour les Langues (CECR). Une combinaison de ces deux oukases est aujourd'hui présente dans tous les manuels de FLE. Puren (2006, p. 40) a expliqué clairement la différence entre ces deux approches pourtant très similaires: si dans l'approche communicative c'est la parole qui est à la fois le moyen et l'objectif, dans l'approche actionnelle elle n'est qu'un moyen au service d'une fin (i.e. une action à mener ensemble). La théorie sous-jacente à ces deux approches est cependant commune, c'est celle d'un apprenant acteur de sa propre formation grâce aux interactions qu'il opère avec un environnement déterminé. Nous reconnaissons là la devise du constructivisme. Et c'est aussi celle de la grande majorité des $\mathrm{MOOC}$, les XMOOC, qui sont proches d'un enseignement traditionnel, entre behaviourisme et constructivisme. Dans les CMOOC au contraire, ce sont les relations entre pairs qui priment, qu'il s'agisse de contenus, de motivation ou d'évaluation. George Siemens (2012) explique ainsi ce qui différencie ces deux types de MOOC: les cMOOC se focalisent sur la création et la production de la connaissance alors que les XMOOC se focalisent sur sa duplication. Les CMOOC se veulent une mise en pratique de ce que Siemens (2005) a appelé le connectivisme. II s'agit d'une théorie de l'apprentissage, née des nouvelles pratiques sociales de loisirs, de communication et d'acquisition de biens culturels. Quand la prégnance de ces pratiques est telle qu'elle ne peut plus être laissée à l'entrée des salles de classe, le plus raisonnable est alors, non seulement d'en tenir compte, mais d'en exploiter les possibilités formatives. C'est ce que propose le connectivisme qui ne peut se définir comme une approche parmi d'autres, ou alors seulement comme une "approche de 
rupture" en donnant à cette expression le sens que Clayton Christensen donne au concept $d^{\prime}$ "innovation de rupture" (disruptive innovation ${ }^{7}$ ). En effet, en tant qu'enseignants, nous sommes hélas habitués à changer de méthodes tous les dix ou vingt ans. Ces changements, terminologiques (apprenant/appreneur) ou architecturaux (tables alignées/tables en $U$ ), saupoudrés çà et là de légers soubresauts méthodologiques étaient loin d'introduire le péril en la demeure. Il en va bien autrement du connectivisme, qui, comme son nom l'indique, insiste avant tout sur l'importance des liaisons établies entre les différentes sources d'information et les différents acteurs du processus d'apprentissage, y compris les liaisons que le web 2.0 suggèrent et génèrent spontanément entre ces informations elles-mêmes, ainsi que les liaisons non recherchées issues de la sérendipité (Serres, 2012, p. 43), qui est l'art de trouver ce que l'on ne cherchait pas. L'unité de base du connectivisme n'est ni la salle de classe, ni le chapitre à étudier, mais les réseaux d'informations générés par les différents acteurs de l'équation pédagogique. Les cours en ligne et les environnements virtuel d'apprentissage avaient donné naissance, il y a quelques années seulement, aux "grains de savoir", ces micros objets pédagogiques, empaquetés à l'identique au format $\mathrm{SCORM}^{8}$ (Sharable Content Object Reference Model) afin d'être réutilisables sur différentes plateformes éducatives comme Moodle, Dokeos, Sakai ou Claroline. Ce que propose le connectivisme en fait d'objets pédagogiques est tout à fait différent: ce ne sont plus des contenus scellés et empaquetés mais une expérience à la fois individuelle et perméable aux informations venues de l'extérieur. Après une décade d'activité, l'épopée SCORM semble être arrivée à son terme et est en passe d'être remplacée par Tin Can $\mathrm{Api}^{9}$, un format qui prendra justement en compte la flexibilité qui manquait au format SCORM. On est passé du grain de savoir, petite unité close sur elle-même et donc facilement maitrisable, au réseau ouvert, de taille variable et donc absolument incontrôlable. En résumé, le connectivisme est une théorie de l'apprentissage qui intègre les principes explorés par le chaos, les réseaux, la complexité et l'auto-organisation (Siemens 2005). On s'aperçoit alors du fossé qui sépare l'approche communicative/actionnelle qui est celle des manuels de langue (qu'ils proposent on non des ressources en ligne) des cMOOC. Certains ont cru reconnaître dans le connectivisme un néosocioconstructivisme (Guité, 2010). Est-ce là le signe d'une impossibilité de penser la nouveauté ou s'agit-il d'une critique pertinente? La notion de paradigme nous

\footnotetext{
7 Voir <http://www.christenseninstitute.org/key-concepts/disruptive-innovation-2/> et la vidéo de Christensen, Disruptive Innovation Explained, <https://www.youtube.com/watch?v= qDrMAzCHFUU> [19/01/2016].

8 SCORM expliqué: <http://scorm.com/scorm-explained/one-minute-scorm-overview/> [21/01/ 2016].

9 Pour en savoir plus sur TinCanApi: < https://tincanapi.com/overview/> [21/01/2016].
} 
donnera des éléments de réponse, mais voyons tout d'abord ce que signifie intégrer la pensée complexe dans une théorie de l'apprentissage.

Le premier et immense avantage qu'apporte le concept de complexité est d'intégrer les contradictions dans l'équation pédagogique afin de donner du réel une vision plus complète. Le second avantage est d'intégrer l'interdisciplinarité, en tant que pratique cognitive usuelle. La pédagogie du Xxl ${ }^{\mathrm{e}}$ siècle sera complexe ou ne sera pas, pour paraphraser un mot d'ordre surréaliste. Elle abandonnera la pensée binaire pour une pensée complexe, pour une pensée "qui relie les connaissances éparses de multiples disciplines, les organise et affronte les contradictions" (Morin, 2015, p. 23). Pour Morin, la réduction du complexe au simple (i.e. du réel de la classe à sa réduction binaire appelée pédagogie) et la réduction du composite en ses éléments (i.e. de l'éducation à ses disciplines académiques) sont toutes deux sources d'erreurs. Un exemple familier de pensée réductionniste appliquée à la pédagogie? La "pratique jivaro" (Closets, 1996, p. 47) de la fiche de lecture, activité sensée éveiller à la littérature mais qui, en pratique, n'en propose qu'une réduction dont tout le charme a disparu. La pédagogie du XXI ${ }^{\mathrm{e}}$ siècle devra abandonner le paradigme cartésien des certitudes bipolaires comme esprit/matière ou quantité/qualité souligne Morin (2000, p. 9) et c'est à l'éducation qu'il échoit la tâche de promouvoir une intelligence générale apte à se référer au complexe. Serres (2012, p. 69) nous rappelle qu'en histoire des sciences la "complexité apparait comme un signe que l'on n'utilise pas la bonne méthode et qu'il faut changer de paradigme". II y a bien en effet un lien entre la pensée complexe et le concept scientifique de paradigme.

Mais qu'est-ce qu'un paradigme? Thomas S. Kuhn en donne la définition épistémologique en 1962. Les paradigmes correspondent à "des vérités scientifiques universellement reconnues qui, pour un temps, fournissent à une communauté de chercheurs des problèmes types et des solutions" (Kuhn, 2008, p. 11). Le mode de connaissance dominant est donc "commandé par un paradigme (principe qui commande l'organisation de la connaissance) contraignant à la disjonction et à la réduction" (Morin, 2015, p. 43) ce qui a pour résultat d'empêcher d'appréhender la complexité du réel. En lieu et place de "disjonction" et de "réduction", le pédagogue lira "étanchéité des disciplines" et "pensée binaire". Depuis les années 1980 et jusqu'à aujourd'hui, I'hymne constructiviste des sciences de l'éducation, repris en chœur par des milliers de praticiens, n'a pas changé: l'apprenant placé au centre du processus d'apprentissage doit être l'acteur de sa propre formation. Notons au passage qu'il ne s'agissait pas même d'une nouveauté puisque déjà en 1932 Alain disait des élèves qu'il fallait "mettre en leurs mains leur propre apprentissage (1976, p. 8) et qu'en 1946 Freinet insistait, lui aussi, sur l'importance de "placer l'enfant au centre des préoccupations pédagogiques" (1978, p. 191). Face au constructivisme, seuls quelques pédagogues ont pris en considération la notion de complexité. C'est le cas 
de Philippe Perrenoud (1993) pour qui reconnaitre la complexité c'est "accepter surtout de changer périodiquement de paradigme" et de Christian Puren (2013) qui a décrit les neuf composantes de la complexité en didactique des langues-cultures. Mais une hirondelle ne fait pas le printemps et il nous faut faire ce constat: depuis près d'un siècle il n'y a pas eu, en pédagogie, d'évolution majeure. Le constructivisme, en tant que paradigme des sciences de l'éducation, a rempli son devoir puisqu'il a fourni, pour un temps, des pistes intéressantes et permis un questionnement utile. En 2016 cependant, il est passé du statut de théorie innovatrice à un précepte de simple bon sens. Un constructivisme de façade, rarement remis en question (voir à ce propos Boghossian 2009) est devenu une "science normale", et la caractéristique de la science normale est de supprimer les nouveautés fondamentales quand elles sont propres à ébranler ses convictions de base (Kuhn, 2008, p. 22). Seul est réel ce qui est quantifiable et il en est ainsi depuis Galilée. Le quantifiable est encore, en ce début du $\mathrm{XXI}^{\mathrm{e}}$ siècle, la base de tout le système éducatif alors que de nouveaux paramètres ont radicalement changé l'équation pédagogique, où l'énigme pédagogique comme dirait Kuhn. Ces nouveaux paramètres ne sont pas pris en compte car les systèmes tendent à négliger ce qu'ils ne peuvent évaluer. Le constructivisme qui a permis bien des avancées en son temps est désormais un paradigme qui, comme tous les paradigmes,

peut tenir le groupe de chercheurs à l'écart des problèmes qui ont leur importance sociale mais ne sont pas réductibles aux données d'une énigme parce qu'ils ne se posent pas en termes compatibles avec les outils conceptuels et instrumentaux que fournit le paradigme. (Kuhn, 2008, p. 63)

Que signifierait alors changer de paradigme? Cela signifierait passer de Galilée et du tout quantifiable, c'est-à-dire d'un système fermé, aux systèmes vivants, aux systèmes ouverts étudiés par von Bertanlaffy et la prise en compte, dans l'étude d'un phénomène, de l'ensemble des variables possibles, des interactions produites et des principes de l'auto-organisation. La définition de l'organisme de von Bertanlaffy (1961, p. 29) appliquée à la classe représenterait celle-ci comme "un système, terme par lequel nous entendons un complexe d'éléments en interaction". Pour donner un exemple pratique, changer de paradigme signifierait prendre en compte dans l'évaluation des étudiants le qualitatif autant que le quantitatif, la cohérence d'une idée ou d'une méthode autant que les contradictions internes surgies de cette même idée, et surtout, analyser la qualité des réseaux d'interactions générés par cet apprenant lors du processus d'acquisition et de création des connaissances.

\subsection{Le savoir libéré}

Libérer le savoir signifie à la fois en libérer l'accès et en libérer la forme. L'accès au savoir se réfère à la disponibilité des sources de la connaissance tandis que les formes 
du savoir renvoient à la multiplicité des réseaux qui le construisent. Nous allons à présent voir de quelle manière pédagogie complexe et libération du savoir vont de pair.

Si les initiatives comme celle de Salman Khan et la profusion des cours en ligne on fait couler beaucoup d'encre, c'est que la technologie 2.0 a permis la réalisation à grande échelle de visions éducatives bien connues, mais dont on s'était fort peu inquiété jusqu'à présent. Les communautés d'experts d'lllich par exemple n'étaient pas matériellement réalisables à grande échelle, à une échelle justement massive, car elles manquaient de l'appui technologique adéquat. L'objectif premier des pédagogues-visionnaires du siècle dernier comme Alain, Freinet et Illich, pour ne citer qu'eux, était d'offrir à toute personne désireuse d'apprendre le libre accès à tout ce qui pourrait l'aider dans l'acquisition de la connaissance voulue. Être cultivé dit Alain "c'est en chaque ordre remonter à la source et boire dans le creux de sa main, non point dans une coupe empruntée" (1976, p. 114) ce qui signifie, par exemple, pour un doctorant, pouvoir accéder aux textes fondateurs, textes qui le plus souvent ne sont disponibles que dans quelques rares bibliothèques, à Paris généralement, et parfois uniquement consultables sur place. Dans les institutions éducatives d'avant l'ère Internet, seul l'enseignant avait accès aux sources vives de la connaissance grâce à son statut, ses revenus et son âge. Pour l'étudiant, le jeune chercheur, le chercheur délocalisé, loin des bibliothèques de référence, l'accès à des savoirs de première main est une course d'obstacles, ardue et onéreuse. Un exemple éloquent de l'enfermement du savoir est celui des conditions d'accès à la Bibliothèque Nationale de France (BNF). Les conditions requises pour accéder aux bibliothèques de recherche sont d'avoir plus de 18 ans et de justifier d'une recherche d'ordre universitaire, professionnel, ou personnel. Une accréditation ${ }^{10}$ délivrée à la suite d'un entretien individuel avec un bibliothécaire, est obligatoire. Nous sommes là aux antipodes de la culture des MOOC. Peut-être est-ce parce que "le démantèlement de l'institution scolaire [...] passe par la promulgation de lois interdisant toute discrimination à l'entrée des centres d'études" (Illich, 1971, p. 28) que fleurissent les empêchements de toutes sortes. Depuis longtemps la véritable autorité de l'enseignant n'est plus celle de la baguette; elle a été remplacée par l'accès privilégié au savoir. Car seul l'enseignant a accès aux sources vives de la connaissance. Que les têtes soient levées vers lui, quand il dispensait son savoir du haut de son estrade, ou qu'elles risquent le torticolis, dans une salle disposée en $U$, l'enseignant est toujours le seul à avoir accès au livre. Le livre avec les corrigés, le livre avec l'information vitale, le livre depuis longtemps épuisé et disponible seulement dans une poignée de bibliothèques. Les collectifs anti-MOOC se souviennent sans doute des propos du

10 Particulièrement édifiant est le formulaire à remplir en vue de préparer ledit entretien: <http://accreditation.bnf.fr/jsp/preinscription.jsp> [22/01/2016]. 
visionnaire de Cuernavaca et en concluent que la non-discrimination à l'entrée des centres d'études impliquera, à court ou à long terme, le démantèlement de l'institution scolaire. Grâce au projet Gutenberg, à Gallica, les livres se sont évadés de leurs îles solitaires et naviguent à l'encontre de leurs lecteurs. Si le doctorant ne trouve pas toujours l'appui nécessaire auprès de son directeur de thèse, il sera certain du moins de ne jamais être déçu par GAFA ${ }^{11}$, son Cicéron virtuel. N'est-il pas révélateur que parmi les plus grands fournisseurs de cours en ligne il y ait Telefónica, une compagnie de télécommunication?

Ce ne sont pas seulement les textes qui sont libérés de leurs étagères poussiéreuses mais tout ce qui permet l'acquisition du savoir: "pour qu'un homme puisse grandir, ce dont il a besoin c'est du libre accès aux choses, aux lieux, aux méthodes, aux événements, aux documents" (Illich, 1971, p. 213). Le libre accès aux choses est même, selon Illich, le premier des quatre principes de la révolution éducative (p. 170). Les canaux traditionnels du savoir que sont les universités et les bibliothèques doivent être doublés de canaux moins rigides, plus flexibles, donc plus efficaces. L'heure est à l'émancipation du savoir de ses canaux de transmissions traditionnels car il faut "donner à celui qui veut apprendre des moyens nouveaux d'entrer en contact avec le monde autour de lui, au lieu de continuer à avoir recours aux canaux de distribution traditionnels des programmes d'enseignement" (Illich, 1971, p. 124). Or souligne Closets voilà "justement ce que l'école ne pourra jamais comprendre: une acquisition qui n'emprunte pas le canal pédagogique traditionnel" (1996, p. 68). Libérer le savoir signifie donc en rendre disponible gratuitement les textes, documents, choses, méthodes et évènements, à tout moment du jour ou de la nuit, à tous les usagers, sans distinction de diplômes, de références fournies ou d'études préalables. Et c'est à ce changement radical du comment enseigner, qui ne se contente pas d'un maquillage terminologique ou architectural superficiel, que se réfère Illich quand il prévoit un changement de paradigme (notons qu'il cite deux fois Kuhn: p. 90 et p. 166). Pour lui, en effet, la formation institutionnalisée est à bout de souffle et son épuisement, comme ses contradictions, précèdent l'apparition d'un nouveau paradigme du savoir.

Assurer l'accès aux savoirs ne signifie pas seulement pour l'enseignant mettre à disposition des apprenants ses notes ou ses documents. L'accès aux savoirs signifie aussi libérer le partage des compétences selon le deuxième principe de la révolution pédagogique d'lllich. En reprenant la différence que fait Alain entre apprendre par l'objet et apprendre par l'esprit nous pouvons dire avec l'auteur des Propos sur l'éducation que "le premier chemin est celui des techniques" (1976, p. 68) et ajouter que le deuxième chemin est celui des technologies 2.0. Le mot n'est bien sûr pas cité

11 GAFA: Google, Apple, Facebook, Amazon. 
par Alain mais il en donne la définition car apprendre par l'esprit c'est apprendre au moyen des interactions entre notre savoir et celui des autres, c'est, selon les mots du philosophe, "faire société" (p. 69). Ce que décrivait ainsi Alain il y a plus de 80 ans, cet apprentissage par l'esprit caractérisé par les interactions et les échanges entre les membres de la communauté éducative, est le principe même des cMOOC. Faire société c'est aussi, aujourd'hui, retweeter car "rebondir sur les autres, avec eux, parmi eux, est l'essence du comportement numérique au quotidien " (Fogel et Patino, 2014, p. 55). Le partage des compétences est à la base des communautés d'experts et c'est une fois de plus chez Illich que nous trouvons une très actuelle description de l'objectif des MOOC: "donner à celui qui veut apprendre des moyens nouveaux d'entrer en contact avec le monde autour de lui, au lieu de continuer à avoir recours aux canaux de distribution traditionnels des programmes d'enseignement" (1971, p. 124). Le concept de serendipité devient alors une des caractéristiques de ces communautés et vient entériner le fait que "ce que l'on a appris vous est souvent venu comme par aventure" (Illich, 1971, p. 30). Il en résulte que le partage des compétences préconisé par Illich et réalisé dans les CMOOC est tout le contraire de l'approche par compétences que décrit Beacco (2007). En effet, "la réception d'un texte n'est pas indifférente à la forme qui l'abrite" (Fogel et Patino, 2014, p. 110) et comme le souligne Beacco lui-même: "il semble difficile d'appréhender les méthodologies d'enseignement en dehors de leurs modes de constitution, de leurs modes de diffusion" (2007, p. 40). Le manuel scolaire, version papier, suit une progression linéaire et sa transposition numérique ne déroge pas à ce mode de présentation malgré quelques média incrustés. C'est ainsi que le changement de paradigme amorcé dans les MOOC est bloqué au niveau des salles de classe. L'approche par compétences qui s'est généralisée dans l'enseignement des langues étrangère se refuse explicitement à sortir du constructivisme, non pour des raisons scientifiques, mais à cause de considérations purement éditoriales car "il s'est avéré que l'approche par compétences constitue un instrument particulièrement adéquat pour la conception de l'architecture des manuels et pour celle de la structuration des séquences d'enseignement" (Beacco, 2007, p. 14). C'est donc le mode de diffusion dominant (papier) et une commode division des unités didactiques qui donnent le ton en pédagogie. Le marché éditorial entrave la réflexion au lieu de la stimuler. Or ce que nous a appris la pensée complexe, c'est que le tout a des propriétés autres que la somme des parties, c'est qu'il ne faut pas s'en tenir aux seuls éléments car cela empêcherait de voir l'harmonie de l'ensemble. De la même façon, il ne faut pas penser "par chapitres, comme tant d'anciens écoliers chez qui la classification arbitraire a tué tout ordre véritable" (Freinet, 1978, p. 45), mais penser avec tout son être car c'est l'être entier qui participe au cheminement de l'esprit. Aux cheminements linéaires, la pensée complexe préfère la boucle et, à la pensée binaire, elle substitue la reliance (Morin, 2015, p. 86). La reliance n'est pas un nouveau dogme, elle n'explique pas tout, elle laisse affleurer les contradictions entre les éléments qu'elle relie 
pourtant. Relier, relier toujours, est pour l'auteur de La Méthode le maître mot éthique et politique. À ce stade de la réflexion nous voyons bien ce qui unit la reliance au connectivisme: c'est l'importance reconnue des liaisons et la bienvenue donnée à des connaissances dé-compartimentées. Le connectivisme peut être alors compris comme le résultat du concept de reliance appliqué au domaine de l'enseignementapprentissage. Pour Morin, la relation entre le je et le nous est "complémentaire, concurrente et antagoniste, c'est-à-dire complexe" (2015, p. 105) et la mission de I'humanisme régénéré qu'il appelle de ses vœux est justement de reconnaitre la complexité humaine, de reconnaitre les contradictions qui fondent l'homo complexus. Cela sera possible si nous abandonnons la rationalité close pour une rationalité ouverte, c'est-à-dire "une manière de penser qui rationnelle qui reconnaît les limites de la raison, affronte les contradictions, et qui est ouverte sur ce qui est réputé irrationnel ou a-rationnel" (Morin, 2015, p. 129). Cette rationalité ouverte est une rationalité complexe qui prend en compte les tensions existantes entre le je et le nous, dans ce cas, entre le rationalisme cartésien et l'irrationalité. Pour les sciences et les humanités, adopter une rationalité ouverte, signifie changer de paradigme ${ }^{12}$ nous dit Morin. La relation paradigmatique nouvelle n'oppose plus dos-à-dos le rationnel à l'irrationnel mais les conjugue au sein d'un paradigme complexe. Elle abandonne donc la pensée binaire. Et c'est là que nous retrouvons notre salle de classe. Ce que Morin dit de l'essoufflement du paradigme cartésien peut être repris à propos de l'éreintement du paradigme constructiviste. Ce qu'il dit d'un possible humanisme régénéré nous le disons d'un possible enseignement régénéré. Le connectivisme, encore à ses débuts, est cependant déjà prêt à affronter la complexité des processus d'enseignement-apprentissage car il a fait le saut qualitatif indispensable qui place toute connaissance dans la complexité et la rationalité ouverte.

\section{Conclusion}

Placer le phénomène des MOOC dans un contexte historique nous aide à mieux comprendre ce qui est en jeu. Le traiter comme le dernier phénomène éducatif à la mode c'est peut-être en rester à la superficialité du gadget et manquer la chance d'amorcer, en sciences de l'éducation, un réel débat. La question se pose ainsi: faut-il en finir avec le paradigme constructiviste pour explorer celui de la pensée complexe? La pensée binaire, qui tire les enseignants à hue et à dia, a expulsé de l'énigme pédagogique les doutes et les contradictions, en somme, tout ce qui fait l'apprentissage même. Les méthodes pédagogiques en FLE se suivent en se tournant le dos. On pourrait même penser que le fait d'aller contre la méthode ancienne est le seul propos de la méthode nouvelle. L'enseignant qui enseigne devant une rangée

12 Notons qu'à propos de changement de paradigme Morin cite Serres p. 148 et Kuhn p. 152. 
d'élèves n'est pas forcément pire que l'animateur qui facilite des ressources au milieu d'un cercle d'apprenants. Nous devons quitter le Far West pédagogique où les uns sont toujours les bons et les autres toujours les méchants. C'est pratique, sans aucun doute, mais peu efficace quand il s'agit de salles de classe et non de salles obscures. Le système classe est avant tout un système et, en tant que tel, il est essentiellement complexe. Au-dedans, les contradictions ne doivent ni s'opposer en vaines apories ni se succéder en un interminable et inefficace défilé. La pensée "revolutionnante" (Morin, 2015, p. 29) qui nous vient du monde des sciences, de la cybernétique des années 1970 exactement, a donné naissance à La Méthode et cette méthode, dans un contexte de réseaux électroniques, a permis au connectivisme de faire ses premiers pas. Dans l'enseignement de FLE, un domaine où la guerre des pédagogies a toujours fait rage, ou les approches se distinguent plus par leur strict antagonisme que par leur scientificité, un changement de paradigme permettant de dépasser le mode binaire de l'actuelle réflexion pédagogique est devenu nécessaire si nous voulons que Petite Poucette continue à fréquenter nos universités. Le paradigme de la reliance, qui prend en compte la totalité des facteurs intervenant dans l'enseignement, et non seulement ceux facilement mesurables, permettra de rendre justice à la complexité des apprentissages, qui est aussi la complexité de la vie.

\section{About the author}

Florence Gérard Lojacono enseigne le français langue-culture ainsi que la didactique du FLE à l'Université de Las Palmas de Gran Canaria. Spécialiste des TICE appliquées au FLE, elle a écrit de nombreux articles qui ont pour point commun de remettre l'humain au centre des relations pédagogiques, même, et surtout, quand celles-ci sont hyper connectées.

\section{Article history}

Paper received: $17^{\text {th }}$ November 2015

Paper received in revised form and accepted for publication: $28^{\text {th }}$ January 2016

\section{Références}

Alain. (1976). Propos sur l'éducation (15 éd.). Paris: PUF. 
Baillon, R. (1990). Les consommateurs d'école. Paris: Stock.

Beacco, J.-C. (2007). L'approche par compétences dans l'enseignement des langues. Enseigner à partir du CECR. Paris: Didier.

Bedel, C. (2014). Quelques vérités à rétablir sur les MOOC. Libération, 6 janvier 2014. <http://www.liberation.fr/societe/2014/01/06/quelques-verites-a-retablir-sur-lesmooc_970928> [04/11/2015].

Bertalanffy von, L. (1961). Les problèmes de la vie. Essai sur la pensé biologique moderne. (M. Deutsch, Trad.). Paris: Gallimard. (Euvre originale publiée en 1949).

Boghossian, P. (2009). La peur du savoir. Sur le relativisme et le constructivisme de la connaissance. (Deroy, O. Trad.). Marseille: Éd. Agone. (đFuvre originale publiée en 2006).

Bowen, J. A. (2012). Teaching naked. How moving technology out of your college classroom will improve student learning. San Francisco: Jossey-Bass.

Conseil de l'Europe. (2001). Cadre européen commun de référence pour les langues: apprendre, enseigner, évaluer. Strasbourg: Division des Politiques linguistiques / Éditions Didier. <http://www.coe.int/t/dg4/linguistic/Source/Framework_FR.pdf> [19/01/2016].

CESE. (2015). La pédagogie numérique: un défi pour l'enseignement supérieur. Journal Officiel de la République française. Séance du 24 février 2015.

Clayton Christensen Institute.

$<$ http://www.christenseninstitute.org/keyconcepts [31/01/2016].
Disruptive Innovation. /disruptive-innovation-2/>

Closets de, F. (1996). Le bonheur d'apprendre et comment on l'assassine. Paris: Seuil.

Compagnon, A. (2014). Moocs et vaches à lait. Le Débat, 180, 170-178.

Collectif anti-MOOC. (2013). Mooc: une étape vers la privatisation des cours. Libération, 26 décembre 2013. <http://www.liberation.fr/societe/2013/12/26/mooc-une-etape-vers-laprivatisation-des-cours_969050> [04/11/2015].

Cuq, J.-P., \& Gruca, I. (2002). Cours de didactique du français langue étrangère et seconde. Grenoble: PUG.

Davidenkoff, E. (2014). Le tsunami numérique. Paris: Stock.

Fogel, J.-F., \& Patino, B. (2014). La condition numérique. Comment Internet bouleverse nos vies. Paris: Points.

Freinet, C. (1978). L'éducation du travail (5 $5^{e}$ éd.). Paris: Delachaux et Niestlé.

Galisson, R. (1980). D'hier à aujourd'hui la didactique des langues étrangères. Du structuralisme au fonctionnalisme. Paris: Clé International.

Guerraoui, R. (2014). Pièges à MOOC. Le Monde, 26 juin 2014. <http://binaire.blog.lemonde.fr/ 2014/06/26/pieges-a-mooc/> [04/11/2015].

Guité, F. (2010). Le connectivisme. <http://www.francoisguite.com/2004/12/le-connectivismeneo-socioconstructivisme/> [30/10/2015]. 
Illich, I. (1971). Une société sans école. (G. Durand, Trad.). Paris: Seuil. (ÆEuvre originale publiée en 1970).

Khan, S. (2013). L'éducation réinventée. Une école grande comme le monde. (P. Chambon, Trad.). Paris: JC Lattès. (CEuvre originale publiée en 2012).

Kuhn, T. S. (2008). La structure des révolutions scientifiques ( $2^{\mathrm{e}}$ éd. de 1970). (L. Meyer, Trad.). Paris: Flammarion. (Euvre originale publiée en 1962).

Le Moigne, J.-L. (2012). Les épistémologies constructivistes. Paris: PUF.

Lojacono, F. (2009). Le rôle de l'enseignant: enfin du nouveau? Cuadernos de Lingüística de la Universidad de Puerto Rico, 2(2), 33-39.

Mérieu, P. (1996). Frankenstein pédagogue. Paris: ESF.

OCDE. (2013). Les grandes mutations qui transforment l'éducation 2013. Paris: OCDE.

<http://dx.doi.org/10.1787/trends_edu-2013-fr> [30/10/2015].

Morin, E. (2000). Les sept savoirs nécessaires à l'éducation du futur. Paris: Seuil. http://unesdoc.unesco. org/images/0011/001177/117740fo.pdf [19/01/2016].

Morin, E. (2015). L'aventure de La Méthode suivi de "Pour une rationalité ouverte". Paris: Seuil.

Perrenoud, P. (1993). L'école face à la complexité. <http://www.unige.ch/fapse/SSE/teachers/ perrenoud/php_main/php_1993/1993_01.html> [19/01/2016].

Prost, A. (1985). Éloge des pédagogues. Paris: Seuil.

Puren, C. (2006). De l'approche communicative à la perspective actionnelle. Le Français dans le Monde, 347, 37-40.

Puren, C. (2013). La complexité en didactique des langues-cultures. <http://www.christianpuren.com/biblioth\%C3\%A8que-de-travail/046/> [19/01/2016].

Rapp. L. (2014). Les MOOCs: révolution ou désillusion? Le savoir à l'heure du numérique. Paris: Institut de l'Entreprise. <http://www.institut-entreprise.fr/les-publications/les-moocsrevolution-ou-desillusion > [19/01/2016].

Serres, M. (2012). Petite Poucette. Paris: Le Pommier.

Siemens, G. (2005). Connectivism: A learning theory for the digital age. International Journal of Instructional Technology and Distance Learning, 2(1), 3-10. <http://www.elearnspace.org/Articles/connectivism.htm> [19/01/2016].

Siemens, G. (2012). MOOCs are really a platform. <http://www.elearnspace. org/blog/2012/07/25/moocs-arereally-a-platform/> [19/01/2016].

Zourou, K. (2007). Paradigme(s) émergent(s) autour des apprentissages collectifs médiatisés en langues. Alsic, 10(2), 3-26. < http://alsic.revues.org/688 > [19/01/2016].

Wieruszowski, H. (1966). The Medieval University: Masters, students, learning. Princeton, New Jersey: D. Van Nostrand Compagny Inc. (An Anvil Original). 